\title{
Maximal finite abelian subgroups of $E_{8}$
}

\author{
Cristina Draper \\ Departamento de Matemática Aplicada, Escuela de las Ingenierías, \\ Universidad de Málaga, Ampliación Campus de Teatinos, 29071 Málaga, \\ Spain (cdf@uma.es)
}

\author{
Alberto Elduque \\ Departamento de Matemáticas e Instituto Universitario de Matemáticas \\ y Aplicaciones, Universidad de Zaragoza, 50009 Zaragoza, Spain \\ (elduque@unizar.es)
}

(MS received 20 March 2015; accepted 1 March 2016)

The maximal finite abelian subgroups, up to conjugation, of the simple algebraic group of type $E_{8}$ over an algebraically closed field of characteristic 0 are computed. This is equivalent to the determination of the fine gradings on the simple Lie algebra of type $E_{8}$ with trivial neutral homogeneous component. The Brauer invariant of the irreducible modules for graded semisimple Lie algebras plays a key role.

Keywords: maximal finite abelian subgroups; fine gradings; $E_{8}$

2010 Mathematics subject classification: Primary 17B25

Secondary 17B40; 20G15

\section{Introduction}

A systematic study of the gradings by abelian groups on the simple Lie algebras was initiated by Patera and Zassenhaus in [19]. For the classical simple Lie algebras over an algebraically closed field of characteristic 0 , the fine gradings were classified in [7]; for the exceptional simple algebras they were classified in [3] and [1] for $G_{2}$, in [4] for $F_{4}$ (see also [9] and [2]), and in [5] for $E_{6}$. The recent monograph [10] collects, among other things, all these results and extensions to prime characteristic.

The problem of the classification, up to equivalence, of fine gradings on a simple Lie algebra $\mathfrak{g}$ over an algebraically closed field of characteristic 0 is equivalent to the problem of the classification, up to conjugation, of the maximal quasi-tori (or maximal abelian diagonalizable subgroups) of the group of automorphisms Aut $(\mathfrak{g})$. In particular, the maximal finite abelian subgroups of Aut $(\mathfrak{g})$ are maximal quasitori.

In the case in which $\mathfrak{g}=\mathfrak{e}_{8}$, the simple Lie algebra of type $E_{8}$, whose group of automorphisms is the exceptional simple algebraic group of type $E_{8}$, some fine gradings have been described in $[10, \S 6.6]$. Our goal is to prove that the list of the corresponding maximal quasi-tori of $E_{8}$ contains the whole list of maximal finite abelian subgroups of Aut $\left(\mathfrak{e}_{8}\right)$. To be precise, the main result of this paper may be summarized in the following theorem.

(C) 2017 The Royal Society of Edinburgh 
TheOREM 1.1. Let $\mathbb{F}$ be an algebraically closed field of characteristic 0. Then, up to conjugation, the list of maximal finite abelian subgroups of the exceptional simple Lie group of type $E_{8}$ consists of

(i) four elementary abelian groups, isomorphic to $\mathbb{Z}_{2}^{9}, \mathbb{Z}_{2}^{8}, \mathbb{Z}_{3}^{5}$ and $\mathbb{Z}_{5}^{3}$;

(ii) three more subgroups, isomorphic to $\mathbb{Z}_{6}^{3}, \mathbb{Z}_{4}^{3} \times \mathbb{Z}_{2}^{2}$ and $\mathbb{Z}_{4} \times \mathbb{Z}_{2}^{6}$.

The maximal elementary abelian $p$-subgroups of the algebraic groups have been obtained in [13], so our aim is to show the existence and uniqueness of the subgroups in item (ii) of the theorem.

Very concrete descriptions of the fine gradings induced by the maximal finite abelian subgroups of $\operatorname{Aut}\left(\mathfrak{e}_{8}\right)$, in terms of some non-associative algebras and constructions of $\mathfrak{e}_{8}$ related to them, can be found in $[10, \S 6.6]$. The maximal quasi-torus corresponding to the fine grading by $\mathbb{Z}_{6}^{3}$ appears also in [14], and a specific model, not relying on non-associative algebras, for the $\mathbb{Z}_{5}^{3}$-grading appears in [6].

A related problem is considered in [21], which studies the abelian subgroups $F$ of the compact (real) simple Lie groups of type $E$ satisfying the condition $\operatorname{dim} \mathfrak{g}_{0}^{F}=$

$\operatorname{dim} F$, where $\mathfrak{g}_{0}$ is the Lie algebra of the Lie group and $\mathfrak{g}_{0}^{F}$ is the subalgebra of fixed elements by the action of $F$. This class of abelian subgroups presents nice functorial properties exploited in [20], and it comprises the class of the maximal finite abelian subgroups $(\operatorname{dim} F=0)$. The close relationship between compact Lie groups and complex reductive linear algebraic groups allows one, in principle, to extract from [21] the list of the maximal finite abelian subgroups of any simple linear algebraic group of type $E$ over $\mathbb{C}$, but there are many details to take care of.

Our approach works over arbitrary algebraically closed fields of characteristic 0 and uses recent results on gradings on simple Lie algebras. We believe both approaches have independent interest.

The next section will be devoted to a survey of some results on matrix algebras. This section is necessary because there will appear gradings on subalgebras of type $A$ and $D$ of $\mathfrak{e}_{8}$ that will have to be extended to the whole of $\mathfrak{e}_{8}$, the main obstruction being the so-called Brauer invariant of an irreducible module for a graded semisimple Lie algebra. Section 3 will present some preliminary results on maximal finite quasi-tori on simply connected algebraic groups, showing that any maximal finite quasi-torus $Q$ of $E_{8}=\operatorname{Aut}\left(\mathfrak{e}_{8}\right)$ is either $p$-elementary abelian for $p=2,3$ or 5 , or its exponent is 6 or 4 and it contains a specific automorphism of $\mathfrak{e}_{8}$ of order 6 or 4 (two possibilities here). The following sections will deal with the different possibilities, assuming that $Q$ is not $p$-elementary abelian.

From now on, the ground field $\mathbb{F}$ will be assumed to be algebraically closed of characteristic zero. Unadorned tensor products will indicate products over $\mathbb{F}$.

\section{Background on gradings}

If $A$ is an abelian group, an $A$-grading on a non-associative (i.e. not necessarily associative) algebra $\mathcal{A}$ over $\mathbb{F}$ is a vector space decomposition $\Gamma: \mathcal{A}=\bigoplus_{a \in A} \mathcal{A}_{a}$ such that $\mathcal{A}_{a} \mathcal{A}_{b} \subset \mathcal{A}_{a b}$ for all $a, b \in A$. The subspaces $\mathcal{A}_{a}$ are said to be the homogeneous components of $\Gamma$ and the non-zero elements $x \in \mathcal{A}_{a}$ are called homogeneous of degree $a$; we will write $\operatorname{deg} x=a$. The support of $\Gamma$ is the $\operatorname{set} \operatorname{Supp} \Gamma:=\left\{a \in A: \mathcal{A}_{a} \neq 0\right\}$. 
Given gradings $\Gamma: \mathcal{A}=\bigoplus_{a \in A} \mathcal{A}_{a}$ and $\Gamma^{\prime}: \mathcal{A}=\bigoplus_{b \in B} \mathcal{A}_{b}^{\prime}$, we say that $\Gamma$ is a refinement of $\Gamma^{\prime}$ if for any $a \in A$ there exists $b \in B$ such that $\mathcal{A}_{a} \subset \mathcal{A}_{b}^{\prime}$. A grading $\Gamma$ is said to be fine if it does not admit a proper refinement.

Given a quasi-torus $Q$ of $\operatorname{Aut}(\mathcal{A})$, we can identify $Q$ with the group of characters $\hat{A}$ for $A$ the group of homomorphisms (as algebraic groups) $Q \rightarrow \mathbb{F}^{\times}$. Then $Q$ induces an $A$-grading of $\mathcal{A}$, where $\mathcal{A}_{a}=\{x \in \mathcal{A}: \chi(x)=a(\chi) x \forall \chi \in Q\}$ for any $a \in A$. It turns out [19] that, in this way, the fine gradings on $\mathcal{A}$, up to equivalence, correspond to the conjugacy classes in $\operatorname{Aut}(\mathcal{A})$ of the maximal quasi-tori (or maximal abelian diagonalizable subgroups) of $\operatorname{Aut}(\mathcal{A})$.

Let $\mathfrak{g}$ be a finite-dimensional simple Lie algebra over $\mathbb{F}$ and let $Q$ be a maximal quasi-torus of $\operatorname{Aut}(\mathfrak{g})$. If $Q$ is finite, the neutral homogeneous component of the associated fine grading $\Gamma$ (i.e. the subalgebra of the elements fixed by the automorphisms in $Q$ ) is trivial (see, for instance, [8, proposition 4.1] or [4, corollary 5]).

For $\mathfrak{g}$ the simple special Lie algebra $\mathfrak{s l}(V)$, if the quasi-torus $Q$ (satisfying this last condition on the neutral component) is contained in the connected component $\operatorname{Aut}(\mathfrak{s l}(V))^{\circ} \simeq \operatorname{PSL}(V)$, then the grading on $\mathfrak{s l}(V)$ is the restriction of a grading on the associative algebra $\mathcal{R}=\operatorname{End}_{\mathbb{F}}(V)$ with $\operatorname{dim} \mathcal{R}_{e}=1$. These kinds of gradings on $\mathcal{R}$ are called division gradings (and $\mathcal{R}$ is called a graded division algebra), since every non-zero homogeneous element is invertible. These gradings are described in $[10$, ch. 2$]$, and as a consequence we have the following result.

TheOrem 2.1. Let $Q$ be a quasi-torus of $\operatorname{PSL}(V) \simeq \operatorname{Aut}(\mathfrak{s l}(V))^{\circ}$ such that the neutral component of the induced grading $\Gamma$ on $\mathfrak{s l}(V)$ is trivial. Then there are a decomposition $V=V_{1} \otimes \cdots \otimes V_{r}$ ( $\operatorname{dim} V_{i}=l_{i} \geqslant 2$ for any $i$ ) and elements $x_{i}, y_{i} \in \mathrm{SL}\left(V_{i}\right)$ with $x_{i} y_{i}=\xi_{i} y_{i} x_{i}$, where $\xi_{i}$ is a fixed primitive $l_{i}$ th root of 1 , $x_{i}^{l_{i}}=y_{i}^{l_{i}}=(-1)^{l_{i}+1} 1_{V_{i}}$, such that

$$
Q=\left\langle\left[x_{1}\right],\left[y_{1}\right], \ldots,\left[x_{r}\right],\left[y_{r}\right]\right\rangle \cong \mathbb{Z}_{l_{1}}^{2} \times \cdots \times \mathbb{Z}_{l_{r}}^{2},
$$

where any endomorphism $z_{i} \in \mathrm{GL}\left(V_{i}\right)$ is identified with the endomorphism $1_{V_{1}} \otimes$ $\cdots \otimes z_{i} \otimes \cdots \otimes 1_{V_{l}}$ (Kronecker product) in $\mathrm{GL}(V)$, and where $[z]$ denotes the class of $z \in \mathrm{SL}(V)$ in $\operatorname{PSL}(V)$. If $r=1$, or if the $l_{i} s$ are powers of prime numbers, the elements $x_{i}$ and $y_{i}$ are unique up to simultaneous conjugation.

Every non-zero homogeneous component of $\Gamma$ has dimension 1, so that $\Gamma$ is fine.

Any graded matrix algebra $\mathcal{R}=M_{n}(\mathbb{F})$ is isomorphic to $\operatorname{End}_{\mathcal{D}}(W)$, where $\mathcal{D}$ is a graded division algebra and $W$ is a finite-dimensional graded right free module over $\mathcal{D}$. The graded division algebra $\mathcal{D}$ is determined by the isomorphism class of the graded algebra $\mathcal{R}$ and it is denoted by $[\mathcal{R}]$ (so $[\mathcal{R}]=[\mathcal{D}]$ ). Moreover, given two $A$ graded matrix algebras $\mathcal{R}_{1}=\operatorname{End}_{\mathcal{D}_{1}}\left(W_{1}\right)$ and $\mathcal{R}_{2}=\operatorname{End}_{\mathcal{D}_{2}}\left(W_{2}\right)$, the tensor product $\mathcal{R}_{1} \otimes \mathcal{R}_{2}$ is again an $A$-graded matrix algebra and $\left[\mathcal{R}_{1} \otimes \mathcal{R}_{2}\right]$ depends only on $\left[\mathcal{D}_{1}\right]$ and $\left[\mathcal{D}_{2}\right]$. Thus, we obtain an abelian group: the A-graded Brauer group $[11, \S 2]$ (or [12, appendix A]). The behaviour of this group mimics the behaviour of the classical Brauer group (but this latter one is trivial over algebraically closed fields!). In particular, we have $[\mathcal{D}]^{-1}=\left[\mathcal{D}^{\mathrm{op}}\right]$, as $\mathcal{D} \otimes \mathcal{D}^{\mathrm{op}} \cong \operatorname{End}_{\mathbb{F}}(\mathcal{D})$ as graded algebras, and the grading on $\operatorname{End}_{\mathbb{F}}(\mathcal{D})$ is induced by the grading on $\mathcal{D}$.

Now let $V$ be a finite-dimensional irreducible module for a finite-dimensional semisimple $A$-graded Lie algebra $\mathfrak{g}$ such that the image of the group of characters $\hat{A}$ in $\operatorname{Aut}(\mathfrak{g})$ lies in the connected component $\operatorname{Aut}(\mathfrak{g})^{\circ}$. Then $[11, \S 3]$ the matrix algebra 
$\operatorname{End}_{\mathbb{F}}(V)$ is $A$-graded in a unique way satisfying that the associated epimorphism of associative algebras $\rho: U(\mathfrak{g}) \rightarrow \operatorname{End}_{\mathbb{F}}(V)(U(\mathfrak{g})$ denotes the universal enveloping algebra) is a homomorphism of $A$-graded algebras. Hence, $\operatorname{End}_{\mathbb{F}}(V) \cong \operatorname{End}_{\mathcal{D}}(W)$ for some $\mathcal{D}$ and $W$ as above, and we write $\operatorname{Br}(V)=[\mathcal{D}]$. This is called the Brauer invariant of the irreducible $\mathfrak{g}$-module $V$.

If $V$ admits an $A$-grading compatible with the action of $\mathfrak{g}$, then the Brauer invariant of $V$ is trivial (and vice versa). Such $A$-grading on $V$ is unique up to a shift. In particular, its homogeneous components are uniquely determined. Hence, we have the following lemma.

Lemma 2.2. Let $\mathfrak{g}=\bigoplus_{\bar{r} \in \mathbb{Z}_{n}} \mathfrak{g}_{\bar{r}}$ be a $\mathbb{Z}_{n}$-graded finite-dimensional semisimple Lie algebra such that $\mathfrak{g}_{\overline{0}}$ is semisimple and each $\mathfrak{g}_{\bar{r}}, \bar{r} \neq \overline{0}$, is an irreducible module for $\mathfrak{g}_{\overline{0}}$. Then any refinement $\Gamma$ is determined, up to equivalence, by its restriction $\Gamma_{\overline{0}}$ to $\mathfrak{g}_{\overline{0}}$.

Proof. Since each $\mathfrak{g}_{\bar{r}}, \bar{r} \neq \overline{0}$, is irreducible, the restriction $\Gamma_{\bar{r}}$ of $\Gamma$ to $\mathfrak{g}_{\bar{r}}$ is the unique $A$-grading, up to a shift, on $\mathfrak{g}_{\bar{r}}$ compatible with the $A$-grading $\Gamma_{\overline{0}}$ on $\mathfrak{g}_{\overline{0}}$. Hence, the homogeneous components of $\Gamma$ are all uniquely determined by $\Gamma_{\overline{0}}$.

\section{Finite-order automorphisms}

Let $\mathfrak{g}$ be a finite-dimensional simple Lie algebra and let $G=\operatorname{Aut}(\mathfrak{g})$ be its group of automorphisms. Given a subgroup $H$ of $G, C_{G}(H)$ will denote its centralizer in $G$.

Lemma 3.1. If $Q$ is a maximal quasi-torus in $G$, then $Q$ is self-centralizing, that is, $C_{G}(Q)=Q$.

Proof. By maximality, $Q$ is a closed subgroup of the algebraic group $G$. For any $x \in C_{G}(Q)$, let $x=x_{s} x_{n}$ be its Jordan decomposition [15, $\left.\S 15\right]$. Then the closure of the subgroup generated by $Q$ and $x_{s}$ is diagonalizable, so by maximality of $Q, x_{s} \in Q$, and thus the quotient $C_{G}(Q) / Q$ is unipotent, and hence nilpotent $[15, \S 17.5]$. We conclude that $C_{G}(Q)$ is nilpotent, because $Q$ is central in $C_{G}(Q)$. Then [18, III.3.4, proposition 3.6] implies that since $Q$ is reductive, so is $C_{G}(Q)$ and, therefore, $C_{G}(Q)$ is reductive and nilpotent, and hence its connected component satisfies $C_{G}(Q)^{\circ}=Z\left(C_{g}(Q)^{\circ}\right)$, and it consists of semisimple elements. By maximality, $C_{G}(Q)^{\circ}=Q^{\circ}$, so $\left[C_{G}(Q): Q\right] \leqslant\left[C_{G}(Q): C_{G}(Q)^{\circ}\right]<\infty$. Therefore, $C_{G}(Q) / Q$ is unipotent and finite, so it is trivial (we are assuming char $\mathbb{F}=0$ ).

In the case in which $\mathfrak{g}=\mathfrak{e}_{8}$, the group $G$ is connected and simply connected, so the next result applies.

Lemma 3.2. Assume that $G$ is semisimple, connected and simply connected, and let $Q$ be a maximal quasi-torus of $G$ with $Q$ finite. Then for any $\theta \in Q$, the subalgebra of fixed elements $\mathfrak{g}^{\theta}:=\{x \in \mathfrak{g}: \theta(x)=x\}$ is a semisimple subalgebra.

Proof. Since $\theta$ is semisimple (finite order) and $G$ is connected and simply connected, $C_{G}(\theta)$ is reductive [16, theorem 2.2], and since $G$ is simply connected, $C_{G}(\theta)$ is connected [16, theorem 2.11]. Then [16, lemma 19.5] $Z\left(C_{G}(\theta)\right)^{\circ}$ is a torus.

But $Z\left(C_{G}(\theta)\right)^{\circ}$ is contained in any maximal quasi-torus of $C_{G}(\theta)$, so it is contained in $Q$. Since $Q$ is finite, we get $Z\left(C_{G}(\theta)\right)^{\circ}=1$, so $\operatorname{dim} Z\left(C_{G}(\theta)\right)=0$, and 
hence the Lie algebra $\mathcal{L}\left(Z\left(C_{G}(\theta)\right)\right)$ is trivial. It follows that the reductive Lie algebra $\mathfrak{g}^{\theta}=\mathcal{L}\left(C_{G}(\theta)\right)$ has trivial centre, so it is semisimple.

The finite-order automorphisms of $\mathfrak{g}$ are classified, up to conjugation, in $[17, \S 8.6]$ in terms of affine Dynkin diagrams and sequences of relatively prime non-negative integers $\left(s_{0}, \ldots, s_{l}\right)$ (here $l+1$ is the number of nodes in the affine Dynkin diagram). For $\mathfrak{g}=\mathfrak{e}_{8}$, if $\theta$ is a non-trivial finite order automorphisms and $\mathfrak{g}^{\theta}$ is semisimple, [17, proposition 8.6] shows that the sequence is of the form $(0, \ldots, 1, \ldots, 0)$ with only one $s_{i}=1$ and $i>0$. That is, only one node is involved in the affine Dynkin diagram $E_{8}^{(1)}$ :

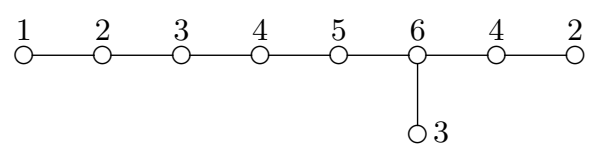

This unique node will be highlighted in black. In particular any such automorphism has order at most 6 , and up to conjugation there are only two order 4 automorphisms and one order 6 automorphism with semisimple $\mathfrak{g}^{\theta}$.

Proposition 3.3. Let $Q$ be a maximal finite abelian subgroup of Aut $\left(\mathfrak{e}_{8}\right)$. Then either $Q$ is $p$-elementary abelian with $p=2,3$ or 5 , or the exponent of $Q$ is either 4 or 6 . Moreover, for any $\theta \in Q$, the fixed subalgebra $\mathfrak{e}_{8}^{\theta}$ is semisimple.

Proof. By lemma 3.2, $\mathfrak{e}_{8}^{\theta}$ is semisimple for any $\theta \in Q$, so the order of $\theta$ is at most 6. The result follows at once.

The maximal elementary abelian subgroups of algebraic groups have been classified in [13]. For Aut $\left(\mathfrak{e}_{8}\right)$ there are only four such subgroups that coincide with their centralizers (and hence they are maximal abelian subgroups). They are isomorphic to $\mathbb{Z}_{2}^{9}, \mathbb{Z}_{2}^{8}, \mathbb{Z}_{3}^{5}$ and $\mathbb{Z}_{5}^{3}$.

Hence we must consider the situation in which the maximal finite abelian subgroup $Q$ of $\operatorname{Aut}\left(\mathfrak{e}_{8}\right)$ contains an automorphism of order 6 or 4 . (It cannot contain both as there are no elements of order 12 in $Q$.)

\section{Order 6 automorphism}

Let $\theta$ be the order 6 automorphism of $\mathfrak{g}=\mathfrak{e}_{8}$ related to the following diagram:

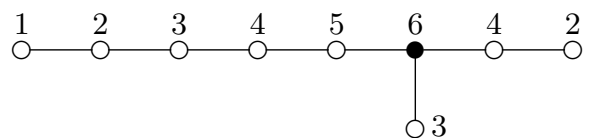

The automorphism $\theta$ induces the $\mathbb{Z}_{6}$-grading $\mathfrak{g}=\bigoplus_{\bar{r} \in \mathbb{Z}_{6}} \mathfrak{g}_{\bar{r}}$, with $\mathfrak{g}_{\bar{r}}=\{x \in$ $\left.\mathfrak{g}: \theta(x)=\xi^{r} x\right\}$, where $\xi$ is a primitive 6 th root of 1 . Then, up to isomorphism, we have

$$
\begin{array}{ll}
\mathfrak{g}_{\overline{0}}=\mathfrak{s l}(U) \oplus \mathfrak{s l}(V) \oplus \mathfrak{s l}(W), & \mathfrak{g}_{\overline{3}}=U \otimes 1 \otimes \wedge^{3} W, \\
\mathfrak{g}_{\overline{1}}=U \otimes V \otimes W, & \mathfrak{g}_{\overline{4}}=1 \otimes V \otimes \wedge^{4} W, \\
\mathfrak{g}_{\overline{2}}=1 \otimes V^{*} \otimes \wedge^{2} W, & \mathfrak{g}_{\overline{5}}=U \otimes V^{*} \otimes \wedge^{5} W,
\end{array}
$$


where $U, V$ and $W$ are vector spaces of dimension 2, 3 and 6 , respectively. The expression above for $\mathfrak{g}_{\bar{r}}, \bar{r} \neq \overline{0}$, gives the structure of $\mathfrak{g}_{\bar{r}}$ as a module for $\mathfrak{g}_{\overline{0}}$. For $\mathfrak{g}_{\overline{0}}$, $\mathfrak{g}_{\overline{1}}$ and $\mathfrak{g}_{\overline{5}}$ this follows from [17, proposition 8.6], as well as the fact that, for $\bar{r} \neq \overline{0}$, $\mathfrak{g}_{\bar{r}}$ is an irreducible module for $\mathfrak{g}_{\overline{0}}$. The other components are computed easily.

In this case, the connected component $\operatorname{Aut}\left(\mathfrak{g}_{\overline{0}}\right)^{\circ}$ is isomorphic to $\operatorname{PSL}(U) \times$ $\operatorname{PSL}(V) \times \operatorname{PSL}(W)$. Moreover, for any $(a, b, c) \in \mathrm{SL}(U) \times \mathrm{SL}(V) \times \operatorname{SL}(W)$, there is an automorphism $\phi_{a, b, c}$ of $\mathfrak{g}$ with

$$
\left.\phi_{a, b, c}\right|_{\mathfrak{g}_{\overline{0}}}=\left(\operatorname{Ad}_{a}, \operatorname{Ad}_{b}, \operatorname{Ad}_{c}\right),\left.\quad \phi_{a, b, c}\right|_{\mathfrak{g}_{\overline{1}}}=a \otimes b \otimes c .
$$

Note that $\mathfrak{g}_{\overline{1}}$ generates $\mathfrak{g}$ as an algebra, so the automorphism $\phi_{a, b, c}$ is determined by its action on $\mathfrak{g}_{\overline{1}}$. We have the following homomorphisms (of algebraic groups):

$$
\begin{aligned}
\Phi: \mathrm{SL}(U) \times \mathrm{SL}(V) \times \mathrm{SL}(W) & \rightarrow C_{G}(\theta), \\
(a, b, c) & \mapsto \phi_{a, b, c}, \\
\Psi: C_{G}(\theta) & \rightarrow \operatorname{Aut}\left(\mathfrak{g}_{\overline{0}}\right), \\
\varphi & \left.\mapsto \varphi\right|_{\mathfrak{g}_{\overline{0}}} .
\end{aligned}
$$

Lemma 4.1.

(i) $\operatorname{im} \Psi=\operatorname{Aut}\left(\mathfrak{g}_{\overline{0}}\right)^{\circ} \simeq \operatorname{PSL}(U) \times \operatorname{PSL}(V) \times \operatorname{PSL}(W)$ and $\operatorname{ker} \Psi=\langle\theta\rangle$ (the subgroup generated by $\left.\theta=\phi_{1_{U}, 1_{V}, \xi 1_{W}}\right)$.

(ii) $\Phi$ is surjective and $\operatorname{ker} \Phi=\left\langle\left(-1_{U}, \xi^{2} 1_{V}, \xi 1_{W}\right)\right\rangle$, which is a cyclic group of order 6 .

Proof. If $\varphi$ is in $\operatorname{ker} \Psi$, then $\left.\varphi\right|_{\mathfrak{g}_{\overline{0}}}=\mathrm{id}$, so by Schur's lemma, $\left.\varphi\right|_{\mathfrak{g}_{\overline{1}}}=\lambda$ id for a nonzero scalar $\lambda$. But $\mathfrak{g}_{\overline{1}}$ generates $\mathfrak{g}$ and this forces $\lambda^{6}=1$, and hence $\varphi$ is a power of $\theta$.

Since $C_{G}(\theta)$ is connected (proof of lemma 3.2), $\operatorname{im} \Psi$ is contained in $\operatorname{Aut}\left(\mathfrak{g}_{\overline{0}}\right)^{\circ}$, so for any $\varphi \in C_{G}(\theta)$ there are elements $a \in \mathrm{SL}(U), b \in \mathrm{SL}(V)$ and $c \in \mathrm{SL}(W)$ such that $\left.\varphi\right|_{\mathfrak{g}_{\overline{0}}}=\left(\operatorname{Ad}_{a}, \operatorname{Ad}_{b}, \operatorname{Ad}_{c}\right)$. But $\phi_{a, b, c} \in C_{G}(\theta)$, and $\Psi(\varphi)=\Psi\left(\phi_{a, b, c}\right)$. Hence $\varphi \phi_{a, b, c}^{-1} \in \operatorname{ker} \Psi=\langle\theta\rangle \subseteq \operatorname{im} \Phi$. Thus, $\varphi \in \operatorname{im} \Phi$ and $\Phi$ is onto.

But $\Psi(\operatorname{im} \Phi)$ fills $\operatorname{Aut}\left(\mathfrak{g}_{\overline{0}}\right)^{\circ} \simeq \operatorname{PSL}(U) \times \operatorname{PSL}(V) \times \operatorname{PSL}(W)$, so we obtain that $\operatorname{im} \Psi=\operatorname{Aut}\left(\mathfrak{g}_{\overline{0}}\right)^{\circ}$.

Finally, for any $a \in \mathrm{SL}(U), b \in \mathrm{SL}(V)$ and $c \in \mathrm{SL}(W)$, the automorphism $\phi_{a, b, c}$ is the identity if and only if $a \otimes b \otimes c=\mathrm{id}$ in $\operatorname{End}_{\mathbb{F}}(U \otimes V \otimes W)$, and this happens if and only if there are scalars $\lambda, \mu, \nu \in \mathbb{F}^{\times}$with $\lambda \mu \nu=1$ such that $a=\lambda 1_{U}, b=\mu 1_{V}$ and $c=\nu 1_{W}$ (which implies that $\lambda^{2}=\mu^{3}=\nu^{6}=1$ because the determinant of these endomorphisms is 1$)$. This shows that $\operatorname{ker} \Phi$ is generated by $\left(-1_{U}, \xi^{2} 1_{V}, \xi 1_{W}\right)=\left(\xi^{3} 1_{U}, \xi^{2} 1_{V}, \xi 1_{W}\right)$.

THEOREM 4.2. Let $Q$ be a maximal finite abelian subgroup of $\operatorname{Aut}\left(\mathfrak{e}_{8}\right)$ containing an order 6 automorphism. Then there are $a_{1}, a_{2} \in \mathrm{SL}(U)$ with $a_{1}^{2}=a_{2}^{2}=-1_{U}$, $a_{1} a_{2}=-a_{2} a_{1}, b_{1}, b_{2} \in \mathrm{SL}(V)$ with $b_{1}^{3}=b_{2}^{3}=1_{V}, b_{1} b_{2}=\xi^{2} b_{2} b_{1}$, and $c_{1}, c_{2} \in \mathrm{SL}(W)$ with $c_{1}^{6}=c_{2}^{6}=-1_{W}, c_{1} c_{2}=\xi c_{2} c_{1}$ ( $\xi$ is a primitive 6 th root of 1 ) such that $Q$ is conjugated to

$$
\left\langle\phi_{a_{1}, b_{1}, c_{1}}, \phi_{a_{2}, b_{2}, c_{2}}, \phi_{1_{U}, 1_{V}, \xi 1_{W}}\right\rangle \cong \mathbb{Z}_{6}^{3} .
$$

Therefore, up to conjugation, Aut $\left(\mathfrak{e}_{8}\right)$ contains a unique maximal finite abelian subgroup with elements of order 6. 
Proof. Let $\theta \in Q$ be an automorphism of order 6 as above. The quasi-torus $Q \subset$ $C_{G}(\theta)$ induces a fine grading $\Gamma$ on $\mathfrak{g}$ (a refinement of the $\mathbb{Z}_{6}$-grading) with trivial neutral homogeneous component, which restricts to a grading $\Gamma_{\overline{0}}$ on $\mathfrak{g}_{\overline{0}}$, and hence on $\mathfrak{s l}(U), \mathfrak{s l}(V)$ and $\mathfrak{s l}(W)$ with trivial neutral homogeneous components. Denote by $\pi_{U}, \pi_{V}$ and $\pi_{W}$ the projections of $\operatorname{Aut}\left(\mathfrak{g}_{\overline{0}}\right)^{\circ}$ onto $\operatorname{PSL}(U), \operatorname{PSL}(V)$ and $\operatorname{PSL}(W)$, respectively. In particular, $\pi_{U} \circ \Psi(Q)$ is a diagonalizable subgroup in $\operatorname{PSL}(U)$ whose induced grading on $\mathfrak{s l}(U)$ satisfies that its neutral component is trivial. By theorem 2.1 , the only possibility is that $\pi_{U} \circ \Psi(Q)$ is isomorphic to $\mathbb{Z}_{2}^{2}$. Analogously, $\pi_{V} \circ \Psi(Q) \cong \mathbb{Z}_{3}^{2}$ and $\pi_{W} \circ \Psi(Q) \cong \mathbb{Z}_{6}^{2}$.

This shows, in particular, that there are elements $c_{1}, c_{2} \in \operatorname{SL}(W)$ with $c_{1}^{6}=c_{2}^{6}=$ -1 and $c_{1} c_{2}=\xi c_{2} c_{1}$ such that $\pi_{W} \circ \Psi(Q)=\left\langle\left[c_{1}\right],\left[c_{2}\right]\right\rangle$. Hence there are elements $a_{1}, a_{2} \in \mathrm{SL}(U)$ and $b_{1}, b_{2} \in \mathrm{SL}(V)$ such that $\phi_{a_{1}, b_{1}, c_{1}}$ and $\phi_{a_{2}, b_{2}, c_{2}}$ are in $Q$. Now we get the following.

- Since $\pi_{U} \circ \Psi(Q) \cong \mathbb{Z}_{2}^{2}$, we have $\operatorname{Ad}_{a_{i}}^{2}=\mathrm{id}, i=1,2$, so $a_{i}^{2}=\varepsilon_{i} 1_{U}$, with $\varepsilon_{i}= \pm 1, i=1,2\left(\right.$ as $\left.\operatorname{det}\left(a_{i}\right)=1\right)$.

- Also, $\pi_{V} \circ \Psi(Q) \cong \mathbb{Z}_{3}^{2}$, so $\operatorname{Ad}_{b_{i}}^{3}=1$ and $b_{i}^{3}=\mu_{i} 1_{V}$ with $\mu_{i}^{3}=1, i=1,2$.

- $\phi_{a_{i}, b_{i}, c_{i}}^{6}=\mathrm{id}$, so id $=a_{i}^{6} \otimes b_{i}^{6} \otimes c_{i}^{6}=-a_{i}^{6} \otimes b_{i}^{6} \otimes 1_{W}=\left(-\varepsilon_{i}^{3} \mu_{i}^{2}\right)$ id. Hence $\varepsilon_{i} \mu_{i}^{2}=-1$, and this ensures that $\varepsilon_{i}=-1$ and $\mu_{i}=1, i=1,2$.

- Since $Q$ is abelian, $\phi_{a_{1}, b_{1}, c_{1}} \phi_{a_{2}, b_{2}, c_{2}}=\phi_{a_{2}, b_{2}, c_{2}} \phi_{a_{1}, b_{1}, c_{1}}$, and hence $a_{1} a_{2} \otimes$ $b_{1} b_{2} \otimes c_{1} c_{2}=a_{2} a_{1} \otimes b_{2} b_{1} \otimes c_{2} c_{1}$, that is, $\xi a_{1} a_{2} \otimes b_{1} b_{2}=a_{2} a_{1} \otimes b_{2} b_{1}$. It then follows that there are scalars $\mu, \nu \in \mathbb{F}^{\times}$such that $a_{1} a_{2}=\mu a_{2} a_{1}$ and $b_{1} b_{2}=\nu b_{2} b_{1}$. Besides, $\mu^{2}=1$ (because $\operatorname{det}\left(a_{1} a_{2}\right)=1$ ), $\nu^{3}=1$, and $\xi \mu \nu=1$. We conclude that $a_{1} a_{2}=-a_{2} a_{1}$ and $b_{1} b_{2}=\xi^{2} b_{2} b_{1}$, and hence we obtain $\pi_{U} \circ \Psi(Q)=\left\langle\left[a_{1}\right],\left[a_{2}\right]\right\rangle$ and $\pi_{V} \circ \Psi(Q)=\left\langle\left[b_{1}\right],\left[b_{2}\right]\right\rangle$.

If $\varphi \in Q$, let us check that $\varphi \in\left\langle\phi_{a_{1}, b_{1}, c_{1}}, \phi_{a_{2}, b_{2}, c_{2}}, \theta\right\rangle$. As $\pi_{W} \circ \Psi(\varphi) \in\left\langle\left[c_{1}\right],\left[c_{2}\right]\right\rangle$, there are integers $0 \leqslant n_{1}, n_{2} \leqslant 5$ such that

$$
\varphi \phi_{a_{1}, b_{1}, c_{1}}^{n_{1}} \phi_{a_{2}, b_{2}, c_{2}}^{n_{2}} \in \operatorname{ker}\left(\pi_{W} \circ \Psi\right) .
$$

Hence we may assume that $\varphi$ is in $\operatorname{ker}\left(\pi_{W} \circ \Psi\right)$. Since $\Phi$ is onto, there are elements $a \in \mathrm{SL}(U)$ and $b \in \mathrm{SL}(V)$ with $\varphi=\phi_{a, b, 1_{W}}$. (Note that $\theta=\phi_{1_{U}, 1_{V}, \xi 1_{W}}=$

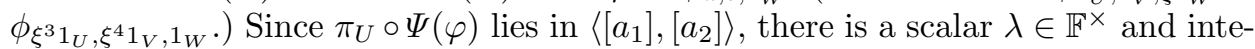
gers $0 \leqslant r_{1}, r_{2} \leqslant 1$ with $a=\lambda a_{1}^{r_{1}} a_{2}^{r_{2}}$. Similarly, there is a scalar $\mu \in \mathbb{F}^{\times}$and integers $0 \leqslant s_{1}, s_{2} \leqslant 2$ with $b=\mu b_{1}^{s_{1}} b_{2}^{s_{2}}$. Also $\lambda^{2}=1=\mu^{3}$ because the determinants are always 1 . Since $\varphi$ commutes with $\phi_{a_{1}, b_{1}, c_{1}}$, we have

$$
\left(a \otimes b \otimes 1_{W}\right)\left(a_{1} \otimes b_{1} \otimes c_{1}\right)=\left(a_{1} \otimes b_{1} \otimes c_{1}\right)\left(a \otimes b \otimes 1_{W}\right),
$$

and, since $a a_{1}=(-1)^{r_{2}} a_{1} a$ and $b b_{1}=\xi^{4 s_{2}} b_{1} b$, we obtain $(-1)^{r_{2}} \xi^{2 s_{2}}=1$, which gives $r_{2}=s_{2}=0$. In the same vein, we get $r_{1}=s_{1}=0$. Therefore, $\varphi \in \operatorname{ker} \Psi=\langle\theta\rangle$, and we obtain $Q=\left\langle\phi_{a_{1}, b_{1}, c_{1}}, \phi_{a_{2}, b_{2}, c_{2}}, \theta\right\rangle$. Moreover, the three generators have order $6, \theta \in \operatorname{ker}\left(\pi_{W} \circ \Psi\right)$ and $\operatorname{im}\left(\left.\pi_{W} \circ \Psi\right|_{Q}\right)=\left\langle\left[c_{1}\right],\left[c_{2}\right]\right\rangle \cong \mathbb{Z}_{6}^{2}$, so $Q$ is isomorphic to $\mathbb{Z}_{6}^{3}$. 


\section{Order 4 automorphism. Type I}

Now let $\theta$ be the order 4 automorphism of $\mathfrak{g}=\mathfrak{e}_{8}$ related to the following diagram:

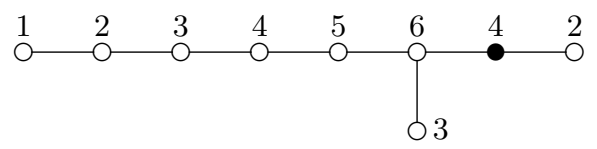

The order 4 automorphisms conjugated to it will be said to be of type $I$. The automorphism $\theta$ induces a grading by $\mathbb{Z}_{4}: \mathfrak{g}=\bigoplus_{\bar{r} \in \mathbb{Z}_{4}} \mathfrak{g}_{\bar{r}}$, with $\mathfrak{g}_{\bar{r}}=\{x \in \mathfrak{g}: \theta(x)=$ $\left.\mathrm{i}^{r} x\right\}$, where $\mathrm{i}$ is a primitive 4 th root of 1 . Then, up to isomorphism, we have

$$
\begin{aligned}
& \mathfrak{g}_{\overline{0}}=\mathfrak{s l}(U) \oplus \mathfrak{s l}(V), \\
& \mathfrak{g}_{\overline{1}}=U \otimes \wedge^{2} V, \\
& \mathfrak{g}_{\overline{2}}=1 \otimes \wedge^{4} V, \\
& \mathfrak{g}_{\overline{3}}=U \otimes \wedge^{6} V,
\end{aligned}
$$

where $U$ and $V$ are vector spaces of dimension 2 and 8 , respectively.

As in the previous section, we have group homomorphisms

$$
\begin{aligned}
\Phi: \mathrm{SL}(U) \times \mathrm{SL}(V) & \rightarrow C_{G}(\theta), \\
(a, b) & \mapsto \phi_{a, b}, \\
\Psi: C_{G}(\theta) & \rightarrow \operatorname{Aut}\left(\mathfrak{g}_{\overline{0}}\right), \\
\varphi & \left.\mapsto \varphi\right|_{\mathfrak{g}_{\overline{0}}},
\end{aligned}
$$

where $\left.\phi_{a, b}\right|_{\mathfrak{g}_{\overline{1}}}=a \otimes \wedge^{2} b$ and $\left.\phi_{a, b}\right|_{\mathfrak{g}_{\overline{0}}}=\left(\operatorname{Ad}_{a}, \operatorname{Ad}_{b}\right)$. The next result is proved along the same lines as lemma 4.1.

LEMMA 5.1.

(i) $\operatorname{im} \Psi=\operatorname{Aut}\left(\mathfrak{g}_{\overline{0}}\right)^{\circ} \simeq \operatorname{PSL}(U) \times \operatorname{PSL}(V)$ and $\operatorname{ker} \Psi=\langle\theta\rangle$.

(ii) $\Phi$ is surjective and $\operatorname{ker} \Phi=\left\langle\left(-1_{U}, \mathrm{i} 1_{V}\right)\right\rangle \cong \mathbb{Z}_{4}$.

THEOREM 5.2. Let $Q$ be a maximal finite abelian subgroup of Aut $\left(\mathfrak{e}_{8}\right)$ containing an order 4 automorphism of type $I$. Then there are $a_{1}, a_{2} \in \mathrm{SL}(U)$ and $b_{1}, b_{2}, c_{1}, c_{2} \in$ $\mathrm{SL}(V)$ with $a_{i}^{2}=-1_{U}$ and $b_{i}^{4}=-1_{V}=c_{i}^{2}$ for any $i=1,2$; also, $a_{1} a_{2}=-a_{2} a_{1}$, $b_{1} b_{2}=\mathrm{i} b_{2} b_{1}, c_{1} c_{2}=-c_{2} c_{1}$ and $b_{i} c_{j}=c_{j} b_{i}$, such that $Q$ is conjugated to

$$
\left\langle\phi_{a_{1}, b_{1}}, \phi_{a_{2}, b_{2}}, \phi_{1_{U}, c_{1}}, \phi_{1_{U}, c_{2}}, \phi_{\mathrm{i} 1_{U}, 1_{V}}\right\rangle .
$$

In particular, up to conjugation, Aut( $\left.\mathfrak{e}_{8}\right)$ contains a unique maximal finite abelian subgroup with automorphisms of order 4 and type $I$, which is isomorphic to $\mathbb{Z}_{4}^{3} \times \mathbb{Z}_{2}^{2}$.

Proof. Let $\theta \in Q$ be an automorphism of order 4 and type I. We can assume that $\theta=\phi_{\mathrm{i}_{U}, 1_{V}}=\phi_{1_{U}, \omega 1_{V}}$, for $\omega$ a primitive eighth root of 1 with $\mathrm{i}=\omega^{2}$.

Denote by $\pi_{U}$ and $\pi_{V}$ the projections of $\operatorname{Aut}\left(\mathfrak{g}_{\overline{0}}\right)^{\circ}$ onto $\operatorname{PSL}(U)$ and $\operatorname{PSL}(V)$, respectively. By theorem 2.1, $\pi_{U} \circ \Psi(Q)$ is necessarily isomorphic to $\mathbb{Z}_{2}^{2}$, while $\pi_{V} \circ$ $\Psi(Q)$, which is a 2 -group of exponent less than or equal to 4 , is isomorphic either to 
$\mathbb{Z}_{4}^{2} \times \mathbb{Z}_{2}^{2}$, or to $\mathbb{Z}_{2}^{6}$. In particular, there are elements $a_{1}, a_{2} \in \operatorname{SL}(U)$ such that $a_{1}^{2}=$ $-1_{U}=a_{2}^{2}, a_{1} a_{2}=-a_{2} a_{1}$ and $\pi_{U} \circ \Psi(Q)=\left\langle\left[a_{1}\right],\left[a_{2}\right]\right\rangle$, and hence there are elements $b_{1}, b_{2} \in \mathrm{SL}(V)$ such that $\phi_{a_{1}, b_{1}}, \phi_{a_{2}, b_{2}}$ are in $Q$. Since both automorphisms commute, $\phi_{a_{1} a_{2}, b_{1} b_{2}}=\phi_{-a_{1} a_{2}, b_{2} b_{1}}$, so that $\wedge^{2}\left(b_{1} b_{2}\right)=-\wedge^{2}\left(b_{2} b_{1}\right)$ and this ensures that $b_{1} b_{2}=$ $\omega^{ \pm 2} b_{2} b_{1}$. Also, $\phi_{a_{i}, b_{i}}^{4}=\mathrm{id}$, so $\operatorname{Ad}_{b_{i}}^{4}=\mathrm{id}, i=1$, 2 . Since $\operatorname{Ad}_{b_{1}}\left(b_{2}\right)=\omega^{ \pm 2} b_{2}$, we obtain that the order of $\operatorname{Ad}_{b_{i}}$ is exactly $4, i=1,2$, and hence both $\left\langle\phi_{a_{1}, b_{1}}, \phi_{a_{2}, b_{2}}\right\rangle$ and $\pi_{V} \circ \Psi\left(\left\langle\phi_{a_{1}, b_{1}}, \phi_{a_{2}, b_{2}}\right\rangle\right)$ are isomorphic to $\mathbb{Z}_{4}^{2}$. Therefore, $\tilde{Q}=\pi_{V} \circ \Psi(Q)$ is necessarily isomorphic to $\mathbb{Z}_{4}^{2} \times \mathbb{Z}_{2}^{2}$. Interchanging the indices if necessary, we may always assume that $b_{1} b_{2}=\omega^{2} b_{2} b_{1}$.

Let us now prove that $\operatorname{ker}\left(\left.\pi_{V} \circ \Psi\right|_{Q}\right)=\langle\theta\rangle$. If $\varphi$ is an element of $Q \cap \operatorname{ker}\left(\pi_{V} \circ \Psi\right)$, then there are elements $a \in \mathrm{SL}(U)$ and $\mu \in \mathbb{F}^{\times}$with $\mu^{8}=1$ (i.e. $\mu 1_{V} \in \mathrm{SL}(V)$ ), such that $\varphi=\phi_{a, \mu 1_{V}}$. But $\varphi \phi_{a_{i}, b_{i}}=\phi_{a_{i}, b_{i}} \varphi$ for $i=1,2$, because $Q$ is abelian, so $a a_{i}=a_{i} a$ for $i=1,2$ and, since $a_{1}$ and $a_{2}$ generate $\operatorname{End}_{\mathbb{F}}(U)$, it follows that $a \in \mathbb{F}^{\times} 1_{U}$. Hence $a= \pm 1_{U}$ and $\varphi \in \operatorname{ker} \Psi=\langle\theta\rangle$.

Therefore, $Q$ is a 2-group of exponent 4 , with $\pi_{V} \circ \Psi(Q) \cong \mathbb{Z}_{4}^{2} \times \mathbb{Z}_{2}^{2}$ and $\operatorname{ker}\left(\pi_{V} \circ\right.$ $\left.\left.\Psi\right|_{Q}\right) \cong \mathbb{Z}_{4}$, implying that $Q$ is isomorphic to $\mathbb{Z}_{4}^{3} \times \mathbb{Z}_{2}^{2}$.

Moreover, the quasi-torus $\tilde{Q} \cong \mathbb{Z}_{4}^{2} \times \mathbb{Z}_{2}^{2}$ induces a division grading on $\operatorname{End}_{\mathbb{F}}(V)$. The elements $b_{1}$ and $b_{2}$ are homogeneous, $\operatorname{alg}\left\langle b_{1}, b_{2}\right\rangle$ is isomorphic to $M_{4}(\mathbb{F})$, and $\operatorname{End}_{\mathbb{F}}(V)=\operatorname{alg}\left\langle b_{1}, b_{2}\right\rangle \otimes C$, where $C$ is the centralizer in $\operatorname{End}_{\mathbb{F}}(V)$ of alg $\left\langle b_{1}, b_{2}\right\rangle$. Thus $C$ is a graded subalgebra of $\operatorname{End}_{\mathbb{F}}(V)$ isomorphic to $M_{2}(\mathbb{F})$. Hence we obtain $V=V_{1} \otimes V_{2}, \operatorname{dim} V_{1}=4, \operatorname{dim} V_{2}=2$, and $\operatorname{alg}\left\langle b_{1}, b_{2}\right\rangle=\operatorname{End}_{\mathbb{F}}\left(V_{1}\right), C=\operatorname{End}_{\mathbb{F}}\left(V_{2}\right)$.

Since $C$ is a graded subalgebra,

$$
\tilde{Q}=\left\langle\left[b_{1}\right],\left[b_{2}\right],\left[c_{1}\right],\left[c_{2}\right]\right\rangle
$$

for elements $c_{i} \in \mathrm{SL}\left(V_{2}\right) \subseteq C, c_{i}^{2}=-1_{V_{2}}, i=1,2$, and $c_{1} c_{2}=-c_{2} c_{1}$. Then there are elements $\hat{a}_{i} \in \mathrm{SL}(U)$ such that $\phi_{\hat{a}_{i}, c_{i}} \in Q, i=1,2$.

The commutativity of $Q$ gives $\phi_{\hat{a}_{i}, c_{i}} \phi_{a_{j}, b_{j}}=\phi_{a_{j}, b_{j}} \phi_{\hat{a}_{i}, c_{i}}$ for any $i, j=1,2$, and since $a_{1}$ and $a_{2}$ generate $\operatorname{End}_{\mathbb{F}}(U)$ and $c_{i} b_{j}=b_{j} c_{i}$, it follows that $\hat{a}_{i} \in \mathbb{F}^{\times} 1_{U}$, so $\hat{a}_{i}= \pm 1_{U}\left(\operatorname{det}\left(\hat{a}_{i}\right)=1\right)$. Composing $\phi_{1_{U}, c_{i}}$ with $\phi_{-1_{U}, 1_{V}}=\phi_{1_{U}, \omega^{2} 1_{V}}=\theta^{2}$ if needed, we may assume $\phi_{1_{U}, c_{i}} \in Q, i=1,2$, and hence the result follows.

\section{Order 4 automorphism. Type II}

Finally, let $\theta$ be the order 4 automorphism of $\mathfrak{g}=\mathfrak{e}_{8}$ related to the following diagram:

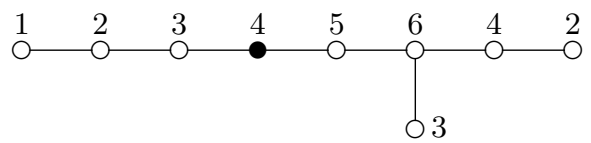

which will be said to be of type II. Thus $\theta$ induces a $\mathbb{Z}_{4}$-grading $\mathfrak{g}=\bigoplus_{\bar{r} \in \mathbb{Z}_{4}} \mathfrak{g}_{\bar{r}}$, where $\mathfrak{g}_{\bar{r}}=\left\{x \in \mathfrak{g}: \theta(x)=\mathrm{i}^{r} x\right\}$, that satisfies

$$
\begin{aligned}
& \mathfrak{g}_{\overline{0}}=\mathfrak{s l}(U) \oplus \mathfrak{s o}(V, q), \\
& \mathfrak{g}_{\overline{1}}=U \otimes V^{+}, \\
& \mathfrak{g}_{\overline{2}}=\wedge^{2} U \otimes V, \\
& \mathfrak{g}_{\overline{3}}=\wedge^{3} U \otimes V^{-},
\end{aligned}
$$


for a four-dimensional vector space $U$ and a ten-dimensional vector space $V$ endowed with a non-degenerate quadratic form $q$. Here $V^{+}$and $V^{-}$denote the two half-spin representations of the orthogonal Lie algebra $\mathfrak{s o}(V, q)$.

Denote by $C(V, q)$ the Clifford algebra of $(V, q)$, and by $x \cdot y$ the multiplication of any two elements $x, y \in C(V, q)$. Recall that $C(V, q)$ is a unital associative algebra generated by $V$ and that $v^{\cdot 2}=q(v) 1$. The Clifford algebra $C(V, q)$ is $\mathbb{Z}_{2}$-graded with $\operatorname{deg} v=\overline{1}$ for any $v \in V$. The spin group is defined as

$$
\operatorname{Spin}(V, q):=\left\{x \in C(V, q)_{\overline{0}}^{\times}: x \cdot V \cdot x^{-1} \subseteq V, x \cdot \varsigma(x)=1\right\},
$$

where $\varsigma$ is the involution (i.e. the antiautomorphism of order 2) of $C(V, q)$ such that $\varsigma(v)=v$ for any $v \in V$.

Let $\left\{e_{1}, \ldots, e_{10}\right\}$ be an orthogonal basis of $V$ with $q\left(e_{i}\right)=1$ for any $i$. Then the centre of $C(V, q)_{\overline{0}}$ is $\mathbb{F} 1 \oplus \mathbb{F} z$, with $z=e_{1} \cdot e_{2} \cdots e_{10} \in \operatorname{Spin}(V, q)$. Moreover, $z^{\cdot 2}=-1$, so the order of $z$ is 4 . There is a surjective homomorphism onto the special orthogonal group

$$
\begin{aligned}
\operatorname{Spin}(V, q) & \rightarrow \mathrm{SO}(V, q), \\
s & \mapsto \iota_{s},
\end{aligned}
$$

where $\iota_{s}(v)=s \cdot v \cdot s^{-1}=s \cdot v \cdot \varsigma(s)$, for any $v \in V$, whose kernel is $\{ \pm 1\}$. Besides, $\iota_{z}=-1_{V}$, so the quotient $\operatorname{Spin}(V, q) /\langle z\rangle$ is isomorphic to the projective special orthogonal group $\operatorname{PSO}(V, q)$, which in turn is naturally isomorphic to the connected component $\operatorname{Aut}(\mathfrak{s o}(V, q))^{\circ}$.

The Lie algebra $\mathfrak{s o}(V, q)$ is isomorphic to the Lie subalgebra

$$
[V, V]^{\cdot}:=\operatorname{span}\left\{[u, v]^{\cdot}=u \cdot v-v \cdot u: u, v \in V\right\}
$$

of $C(V, q)_{\overline{0}}^{-}$. This Lie subalgebra generates $C(V, q)_{\overline{0}}$ (as an associative algebra). The half-spin modules $V^{ \pm}$are the two irreducible modules for the semisimple associative algebra $C(V, q)_{\overline{0}}$ (which are then irreducible modules for $\left.\mathfrak{s o}(V, q) \simeq[V, V]^{\cdot}\right)$. The central element $z$ acts on $V^{+}$(respectively, $V^{-}$) by multiplication by the scalar i (respectively, $-\mathrm{i}$ ).

As for the previous cases, we have a homomorphism

$$
\begin{aligned}
\Phi: \operatorname{SL}(U) \times \operatorname{Spin}(V, q) & \rightarrow C_{G}(\theta), \\
(a, s) & \mapsto \phi_{a, s},
\end{aligned}
$$

such that $\left.\phi_{a, s}\right|_{\mathfrak{g}_{\overline{1}}}$ is given by

$$
\phi_{a, s}(u \otimes x)=a(u) \otimes s . x,
$$

for any $a \in \operatorname{SL}(U), s \in \operatorname{Spin}(V, q), u \in U$ and $x \in V^{+}$, where $s . x$ denotes the action of the element $s \in C(V, q)_{\overline{0}}$ on $x \in V^{+}$. Note that $\mathfrak{g}_{\overline{1}}$ generates $\mathfrak{g}$, so $\phi_{a, s}$ is determined by its action on $\mathfrak{g}_{\overline{1}}$, and $\theta=\phi_{1, z}$. The restriction of $\phi_{a, s}$ to $\mathfrak{g}_{\overline{0}}$ is then given by

$$
\phi_{a, s}(b, \sigma)=\left(\operatorname{Ad}_{a}(b), \operatorname{Ad}_{s}(\sigma)\right),
$$

where $\operatorname{Ad}_{a}(b)=a b a^{-1}$ for $a \in \operatorname{SL}(U)$ and $b \in \mathfrak{s l}(U)$, and $\operatorname{Ad}_{s}(\sigma)=s \cdot \sigma \cdot s^{-1}$ for $s \in \operatorname{Spin}(V, q)$ and $\sigma \in \mathfrak{s o}(V, q) \simeq[V, V]^{\cdot}$ (adjoint action inside $\left.C(V, q)_{\overline{0}}\right)$. Observe that $\operatorname{Ad}_{s}(\sigma)=\iota_{s} \circ \sigma \circ \iota_{s^{-1}}$ for any $s \in \operatorname{Spin}(V, q)$ and $\sigma \in \mathfrak{s o}(V, q) \simeq[V, V]$. 
There is also a group homomorphism:

$$
\begin{aligned}
\Psi: C_{G}(\theta) & \rightarrow \operatorname{Aut}\left(\mathfrak{g}_{\overline{0}}\right), \\
\varphi & \left.\mapsto \varphi\right|_{\mathfrak{g}_{\overline{0}}} .
\end{aligned}
$$

LEMMA 6.1.

(i) $\operatorname{im} \Psi=\operatorname{Aut}\left(\mathfrak{g}_{\overline{0}}\right)^{\circ} \simeq \operatorname{PSL}(U) \times \operatorname{PSO}(V, q)$ and $\operatorname{ker} \Psi=\langle\theta\rangle$.

(ii) $\Phi$ is surjective and $\operatorname{ker} \Phi=\left\langle\left(-\mathrm{i} 1_{U}, z\right)\right\rangle$, which is a cyclic group of order 4 .

Proof. If $\varphi$ is in $\operatorname{ker} \Psi$, then $\left.\varphi\right|_{\mathfrak{g}_{\overline{0}}}=$ id and, by Schur's lemma, $\varphi$ acts as a scalar on $\mathfrak{g}_{\overline{1}}$. This scalar must be a fourth root of 1 and hence $\varphi$ is a power of $\theta$.

For any $\varphi \in C_{G}(\theta)$, the restriction $\left.\varphi\right|_{\mathfrak{g}_{\overline{0}}}$ lies in $\operatorname{Aut}\left(\mathfrak{g}_{\overline{0}}\right)^{\circ} \simeq \operatorname{PSL}(U) \times \operatorname{PSO}(V, q)$ because $C_{G}(\theta)$ is connected (see the proof of lemma 3.2), so there are elements $a \in \mathrm{SL}(U)$ and $s \in \operatorname{Spin}(V, q)$ such that $\left.\varphi\right|_{\mathfrak{g}_{\overline{0}}}=\left.\phi_{a, s}\right|_{\mathfrak{g}_{\overline{0}}}$. Hence $\varphi \phi_{a, s}^{-1} \in\langle\theta\rangle$. But $\theta=\phi_{\mathrm{i} 1_{U}, 1} \in \operatorname{im} \Phi$. It follows that $\Phi$ is onto. Also, $\operatorname{im} \Psi=\Psi(\operatorname{im} \Phi)$ fills $\operatorname{PSL}(U) \times$ $\operatorname{PSO}(V, q) \simeq \operatorname{Aut}\left(\mathfrak{g}_{\overline{0}}\right)^{\circ}$.

Finally, if $\phi_{a, s}=$ id for $a \in \operatorname{SL}(U)$ and $s \in \operatorname{Spin}(V, q)$, then $\operatorname{Ad}_{a}=$ id and $\operatorname{Ad}_{s}=$ id, so $a \in \mathbb{F}^{\times} 1_{U}$ and $s \in Z\left(C(V, q)_{\overline{0}}\right) \cap \operatorname{Spin}(V, q)=\langle z\rangle$. But $\phi_{\lambda 1_{U}, z^{r}}=$ id if and only if $\lambda \mathrm{i}^{r}=1$, or $\lambda=(-\mathrm{i})^{r}$. Then $(a, s)=\left(-\mathrm{i} 1_{U}, z\right)^{r}$, so $\operatorname{ker} \Phi=\left\langle\left(-\mathrm{i} 1_{U}, z\right)\right\rangle$.

LEMmA 6.2. If $\theta$ belongs to a maximal finite abelian subgroup $Q$ of $\operatorname{Aut}\left(\mathfrak{e}_{8}\right)$, then

(a) $Q$ does not contain any element of the form $\phi_{1_{U}, e_{i} \cdot e_{j}}$ with $1 \leqslant i \neq j \leqslant 10$;

(b) if $\phi_{x, s} \in Q$, with $x$ conjugate to

$$
\operatorname{diag}\left\{\omega, \omega^{3}, \omega^{5}, \omega^{7}\right\} \in \operatorname{SL}(U)
$$

and $s \in \operatorname{Spin}(V, q)$, has order 4 and is of type II, then $s^{2}$ has order 4 and is conjugate in $\operatorname{Spin}(V, q)$ to $e_{1} \cdot e_{2} \cdot e_{3} \cdot e_{4} \cdot e_{5} \cdot e_{6}$.

Proof. Any order 4 automorphism of $\mathfrak{e}_{8}$ is of type I if its fixed subalgebra has dimension 66, and of type II if its fixed subalgebra has dimension 60 . In the first case its square is an automorphism of order 2 fixing a subalgebra of type $E_{7}+A_{1}$ and dimension 136, and, in the second one, its square is an automorphism of order 2 fixing a subalgebra of type $D_{8}$ and dimension 120 .

The automorphism $\phi_{1_{U}, e_{i} \cdot e_{j}}$ has order 4 and fixes element-wise a subalgebra isomorphic to $\mathfrak{s l}(U) \oplus \mathfrak{s o}_{2} \oplus \mathfrak{s o}_{8}$ inside $\mathfrak{g}_{\overline{0}}$, and a subspace of the form $\wedge^{2} U \otimes$ $W, \operatorname{dim} W=8$ in $\mathfrak{g}_{2}$. Hence $\operatorname{dim} \mathfrak{g}^{\phi_{1_{U}}, e_{i} \cdot e_{j}} \geqslant(15+1+28)+\left(\begin{array}{l}4 \\ 2\end{array}\right) \times 8=92$. We thus obtain (a).

Note that for any order 2 element in $\mathrm{SO}(V, q)$ there exists an orthogonal basis as above such that this order 2 element is the image of $e_{i_{1}} \cdots e_{i_{2 r}} \in \operatorname{Spin}(V, q)$, for $1 \leqslant i_{1}<\cdots<i_{2 r} \leqslant 10$, and for two commuting order 2 elements in $\operatorname{SO}(V, q)$ there is a common such orthogonal basis.

Since $Q$ has exponent 4, we have $\left(\phi_{x, s}\right)^{4}=\operatorname{id}_{\mathfrak{g}}$ so that $\left(-1, s^{4}\right) \in \operatorname{ker} \Phi=$ $\left\langle\left(-\mathrm{i} 1_{U}, z\right)\right\rangle$ and $s^{4}=-1$. Its square is $\phi_{x^{2}, s^{2}}=\phi_{-\mathrm{i} x^{2}, z s^{2}}$. The order 2 element $z s^{2}$ is then conjugate to either $e_{7} \cdot e_{8} \cdot e_{9} \cdot e_{10}$ or to $e_{3} \cdots \cdot e_{10}$. Now it is easy to compute the dimension of the subspace fixed in each $\mathfrak{g}_{\bar{\imath}}$. The fixed subalgebra of $\phi_{-\mathrm{i} x^{2}, e_{7} \cdot e_{8} \cdot e_{9} \cdot e_{10}}$ has dimension $(7+21)+32+28+32=120$, while the fixed 
subalgebra of $\phi_{-\mathrm{i} x^{2}, e_{3} \cdots \cdots e_{10}}$ has dimension $(7+29)+32+36+32=136$. Hence $z s^{2}$ is conjugate to $e_{7} \cdot e_{8} \cdot e_{9} \cdot e_{10}$ and $s^{2}$ to $e_{1} \cdots e_{6}$.

Denote by $\pi_{U}$ and $\pi_{V}$ the projections of $\operatorname{Aut}\left(\mathfrak{g}_{\overline{0}}\right)^{\circ}$ onto $\operatorname{PSL}(U) \simeq \operatorname{Aut}(\mathfrak{s l}(U))^{\circ}$ and $\operatorname{PSO}(V, q) \simeq \operatorname{Aut}(\mathfrak{s o}(V, q))^{\circ}$, respectively.

Proposition 6.3. If $Q$ is a maximal finite abelian subgroup of $\operatorname{Aut}\left(\mathfrak{e}_{8}\right)$ with automorphisms of order 4 but all of them of type II, then $\pi_{U} \circ \Psi(Q)$ is isomorphic to $\mathbb{Z}_{2}^{4}$ and the grading induced in $\mathfrak{s o}(V, q)$ is elementary, i.e. induced by a grading on $V$.

Proof. Since $\pi_{U} \circ \Psi(Q)$ induces a grading on $\mathfrak{s l}(U)$ such that its neutral component is trivial, we have that theorem 2.1 implies that $\pi_{U} \circ \Psi(Q)$ is isomorphic to either $\mathbb{Z}_{4}^{2}$ or $\mathbb{Z}_{2}^{4}$. Assume that we are in the first case. Then there are $x_{1}, x_{2} \in \operatorname{SL}(U)$ with $x_{1}^{4}=x_{2}^{4}=-1$ and $x_{1} x_{2}=\mathrm{i} x_{2} x_{1}$ such that $\pi_{U} \circ \Psi(Q)=\left\langle\left[x_{1}\right],\left[x_{2}\right]\right\rangle$. Thus there are $s_{1}, s_{2} \in \operatorname{Spin}(V, q)$ with $\phi_{x_{i}, s_{i}} \in Q$. Again by theorem 2.1, $x_{1}$ and $x_{2}$ are, up to simultaneous conjugation,

$$
x_{1}=\operatorname{diag}\left\{\omega, \omega^{3}, \omega^{5}, \omega^{7}\right\}, \quad x_{2}=\left(\begin{array}{cccc}
0 & 0 & 0 & \omega \\
\omega & 0 & 0 & 0 \\
0 & \omega & 0 & 0 \\
0 & 0 & \omega & 0
\end{array}\right) .
$$

By lemma 6.2 we may assume that $s_{1}^{2}=e_{1} \cdot e_{2} \cdot e_{3} \cdot e_{4} \cdot e_{5} \cdot e_{6}$ and, since $s_{1}^{2}$ and $s_{2}^{2}$ anticommute, that either $s_{2}^{2}=e_{4} \cdot e_{5} \cdot e_{6} \cdot e_{7} \cdot e_{8} \cdot e_{9}$ or $s_{2}^{2}=e_{2} \cdot e_{3} \cdot e_{4} \cdot e_{5} \cdot e_{6} \cdot e_{7}$. Lemma 6.1(ii) gives $s_{1} \cdot s_{2}=-z \cdot s_{2} \cdot s_{1}$, and hence $\left(s_{1} \cdot s_{2}\right)^{2}=z \cdot s_{1}^{2} \cdot s_{2}^{2}$ must have order 4 by lemma 6.2(b). But for $s_{2}^{2}=e_{4} \cdot e_{5} \cdot e_{6} \cdot e_{7} \cdot e_{8} \cdot e_{9}$ we get that $\left(s_{1} \cdot s_{2}\right)^{2}=z \cdot s_{1}^{2} \cdot s_{2}^{2}=-z \cdot e_{1} \cdot e_{2} \cdot e_{3} \cdot e_{7} \cdot e_{8} \cdot e_{9}=e_{4} \cdot e_{5} \cdot e_{6} \cdot e_{10}$, which has order 2 , while for $s_{2}^{2}=e_{2} \cdot e_{3} \cdot e_{4} \cdot e_{5} \cdot e_{6} \cdot e_{7},\left(s_{1} \cdot s_{2}\right)^{2}=z \cdot e_{1} \cdot e_{7}$ also has order 2 . In both cases we get a contradiction.

The only possibility, then, is $\pi_{U} \circ \Psi(Q) \cong \mathbb{Z}_{2}^{4}$.

Besides, $\pi_{U} \circ \Psi(Q) \subseteq \operatorname{PSL}(U) \cong \operatorname{Aut}\left(\operatorname{End}_{\mathbb{F}}(U)\right)$ induces a division $\mathbb{Z}_{2}^{4}$-grading on $\operatorname{End}_{\mathbb{F}}(U)=\mathcal{D}$. Therefore, the Brauer invariant of the $\mathfrak{s l}(U)$-module $U$ is $\operatorname{Br}(U)=$ $[\mathcal{D}]$. This allows us to obtain information about the Brauer invariants of the involved $\mathfrak{s o}(V, q)$-modules.

The maximal quasi-torus $Q$ induces a fine grading $\Gamma$ by the group of characters $A$ of $Q$, which is a refinement of the $\mathbb{Z}_{4}$-grading. Thus $\Gamma$ induces a grading $\Gamma_{\overline{0}}$ on $\mathfrak{g}_{\overline{0}}=\mathfrak{s l}(U) \oplus \mathfrak{s o}(V, q)$ by $A$ with support the group of characters of $Q /(Q \cap \operatorname{ker} \Psi)=$ $Q /\langle\theta\rangle$. It also induces a grading $\Gamma_{\bar{r}}$ on each $\mathfrak{g}_{\bar{r}}$ (an irreducible $\mathfrak{g}_{\overline{0}}$-module), $r=1,2,3$, compatible with $\Gamma_{\overline{0}}$. As in lemma 2.2 , up to a shift, $\Gamma_{\bar{r}}$ is the only $A$-grading on $\mathfrak{g}_{\bar{r}}$ compatible with the grading $\Gamma_{\overline{0}}$. Besides, the representation of $\mathfrak{g}_{\overline{0}}$ on $\mathfrak{g}_{\overline{2}}$ gives a graded homomorphism of associative algebras

$$
\rho: U\left(\mathfrak{g}_{\overline{0}}\right) \simeq U(\mathfrak{s l}(U)) \otimes U(\mathfrak{s o}(V, q)) \rightarrow \operatorname{End}_{\mathbb{F}}\left(\mathfrak{g}_{\overline{2}}\right) \simeq \operatorname{End}_{\mathbb{F}}\left(\wedge^{2} U\right) \otimes \operatorname{End}_{\mathbb{F}}(V) .
$$

Then the Brauer invariant of $\mathfrak{g}_{\overline{2}}$, which is trivial since the grading on $\operatorname{End}_{\mathbb{F}}\left(\mathfrak{g}_{2}\right)$ is elementary, satisfies $1=\operatorname{Br}\left(\mathfrak{g}_{\overline{2}}\right)=\operatorname{Br}\left(\wedge^{2} U\right) \operatorname{Br}(V)=[\mathcal{D}]^{2} \operatorname{Br}(V)=\operatorname{Br}(V)$, since $[\mathcal{D}]^{2}=1$. Hence $\operatorname{Br}(V)=1$ and the grading on $\mathfrak{s o}(V, q)$ is induced by a grading on $V$. 
REMARK 6.4. Recall some facts on elementary gradings on simple Lie algebras of type $D_{5}$ from [7] or [10, theorem 3.42]. Let $\tilde{Q}$ be a quasi-torus of $\operatorname{Aut}(\mathfrak{s o}(V, q))$ such that the $A$-grading $\Gamma^{\prime}$ induced on $\mathfrak{s o}(V, q)$ is compatible with a grading on $V$, where $A$ is the group of characters of $\tilde{Q}$. A homogeneous $\mathbb{F}$-basis $\left\{e_{1}, \ldots, e_{10}\right\}$ in $V$ can be selected such that $b$ (the polar form of $q$ ) is represented by the block-diagonal matrix

$$
\operatorname{diag}\left(1, \ldots, 1,\left(\begin{array}{ll}
0 & 1 \\
1 & 0
\end{array}\right), \ldots,\left(\begin{array}{ll}
0 & 1 \\
1 & 0
\end{array}\right)\right)
$$

and the degrees $\operatorname{deg} e_{i}=g_{i}$ satisfy

$$
g_{1}^{2}=\cdots=g_{q}^{2}=g_{q+1} g_{q+2}=\cdots=g_{q+2 s-1} g_{q+2 s}=g_{0}^{-1},
$$

with $q+2 s=10$ and $g_{0} \in A$ is the degree of $b$ (i.e. for any $g, h \in A, b\left(V_{g}, V_{h}\right) \subseteq$ $\left.\mathbb{F}_{g h g_{0}}\right)$. So if $\sigma_{u, v} \in \mathfrak{s o}(V, q)$ denotes the map that sends $w$ to $b(u, w) v-b(v, w) u$, then

$$
\sigma_{e_{q+2 j-1}, e_{q+2 j}} \in(\mathfrak{s o}(V, q))_{g_{q+2 j-1} g_{q+2 j}}=(\mathfrak{s o}(V, q))_{e}
$$

for all $q<j \leqslant 10$. If the grading $\Gamma^{\prime}$ also satisfies that the neutral homogeneous component is trivial, then $q=10$ and it is possible to shift the grading on $V$ to get $g_{0}=e$. To summarize, there is an orthogonal homogeneous basis $\left\{e_{1}, \ldots, e_{10}\right\}$ of $V$ with $q\left(e_{i}\right)=1$ and $\operatorname{deg}\left(e_{i}\right)=g_{i}$ for any $i$, with $g_{i}^{2}=e$ for any $i$. Also, the condition $\mathfrak{s o}(V, q)_{e}=0$ forces all the $g_{i}$ s to be different.

If, in addition, $\tilde{Q}$ is contained in $\operatorname{PSO}(V, q) \simeq \operatorname{Aut}(\mathfrak{s o}(V, q))^{\circ}$, then we have $g_{1} g_{2} \cdots g_{10}=e($ see $[11$, lemma 33]). The elements of $\tilde{Q}$ lift then to $\operatorname{SO}(V, q)$ and they act diagonally in this basis with eigenvalues 1 or -1 . Therefore, we have

$$
\tilde{Q} \subseteq\left\langle\operatorname{Ad}_{e_{i} \cdot e_{j}}: i \neq j\right\rangle
$$

because $\iota_{e_{i} \cdot e_{j}}$ is the diagonal endomorphism with $e_{i}$ and $e_{j}$ eigenvectors with eigenvalue -1 , and $e_{h}$ is fixed by $\iota_{e_{i} \cdot e_{j}}$ for $h \neq i, j$. This means that $\tilde{Q}$ can be embedded in $\mathbb{Z}_{2}^{8}$, since the map

$$
\begin{aligned}
\mathbb{Z}_{2}^{9} & \rightarrow\left\langle\operatorname{Ad}_{e_{i} \cdot e_{j}}: i \neq j\right\rangle, \\
\varepsilon_{i} & \mapsto \operatorname{Ad}_{e_{i} \cdot e_{i+1}},
\end{aligned}
$$

where $\varepsilon_{i}=(\overline{0}, \ldots, \overline{1}, \ldots, \overline{0})(\overline{1}$ in the $i$ th position $)$, is a surjective homomorphism with kernel $\left\langle\varepsilon_{1}+\varepsilon_{3}+\varepsilon_{5}+\varepsilon_{7}+\varepsilon_{9}\right\rangle$, because $e_{1} \cdot e_{2} \cdots e_{10}=z$ and $\operatorname{Ad}_{z}=$ id. Note also that $e_{1} \cdot e_{2} \cdots \cdot e_{8}=-z \cdot e_{9} \cdot e_{10}$, so $\operatorname{Ad}_{e_{1} \cdot e_{2}} \cdots e_{8}=\operatorname{Ad}_{e_{9} \cdot e_{10}}$. In the same vein, $e_{1} \cdot e_{2} \cdots \cdot e_{6}=z \cdot e_{7} \cdot e_{8} \cdot e_{9} \cdot e_{10}$. Thus the group $\left\langle\operatorname{Ad}_{e_{i} \cdot e_{j}}: i \neq j\right\rangle$ consists of the elements id, $\operatorname{Ad}_{e_{i} \cdot e_{j}}$ for $i \neq j$, and $\operatorname{Ad}_{e_{i} \cdot e_{j} \cdot e_{k} \cdot e_{l}}$ for different $i, j, k, l$.

THEOREM 6.5. Up to conjugation, there is a unique maximal finite abelian subgroup $Q$ of Aut $\left(\mathfrak{e}_{8}\right)$ containing an automorphism of order 4 and type II but no automorphism of order 4 and type I. This maximal quasi-torus is isomorphic to $\mathbb{Z}_{4} \times \mathbb{Z}_{2}^{6}$, and a realization is provided by

$$
\left\langle\phi_{x_{1}, e_{8} \cdot e_{9}} \phi_{y_{1}, e_{9} \cdot e_{10}}, \phi_{x_{2}, e_{3} \cdot e_{4}}, \phi_{y_{2}, e_{1} \cdot e_{3} \cdot e_{6} \cdot e_{7}}, \phi_{1_{U}, e_{1} \cdot e_{2} \cdot e_{3} \cdot e_{4}}, \phi_{1_{U}, e_{3} \cdot e_{4} \cdot e_{5} \cdot e_{6}}, \phi_{1_{U}, z}\right\rangle,
$$

for some orthonormal basis $\left\{e_{1}, \ldots, e_{10}\right\}$ relative to $q$. 
Proof. As $\pi_{U} \circ \Psi(Q)$ is isomorphic to $\mathbb{Z}_{2}^{4}$ by proposition 6.3 , we have $\pi_{U} \circ \Psi(Q)=$ $\left\langle\left[x_{1}\right],\left[y_{1}\right],\left[x_{2}\right],\left[y_{2}\right]\right\rangle$ with $x_{i}^{2}=y_{i}^{2}=-1_{U}, x_{i} y_{i}=-y_{i} x_{i}, x_{i} y_{j}=y_{j} x_{i}$, for $i, j \in\{1,2\}$, $i \neq j$, and $x_{1} x_{2}=x_{2} x_{1}, y_{1} y_{2}=y_{2} y_{1}$. Then there are elements $p_{1}, q_{1}, p_{2}, q_{2} \in$ $\operatorname{Spin}(V, q)$ with $\phi_{x_{1}, p_{1}}, \phi_{y_{1}, q_{1}}, \phi_{x_{2}, p_{2}}, \phi_{y_{2}, q_{2}} \in Q$. First, we are going to check that

$$
\left.\operatorname{ker} \pi_{V} \circ \Psi\right|_{Q}=\langle\theta\rangle \text {. }
$$

Take $a \in \operatorname{SL}(U)$ and $s \in \operatorname{Spin}(V, q)$ with $\phi_{a, s} \in Q \cap \operatorname{ker}\left(\pi_{V} \circ \Psi\right)$. $\operatorname{Then} \operatorname{Ad}_{s}=$ id, so $s \in\langle z\rangle$. But $\phi_{-\mathrm{i} 1_{U}, z}=\mathrm{id}$, so if $s=z^{r}, \phi_{a, s}=\phi_{\mathrm{i}^{r} a, 1}$ and we may assume that $s=1$. Now, $\phi_{a, 1}$ is in $Q$, so that $\phi_{a, 1} \phi_{x_{i}, p_{i}}=\phi_{x_{i}, p_{i}} \phi_{a, 1}$ and $\phi_{a, 1} \phi_{y_{i}, q_{i}}=\phi_{q_{i}, y_{i}} \phi_{a, 1}$. Hence $a$ commutes with $\left\{x_{1}, x_{2}, y_{1}, y_{2}\right\}$, which generates $\operatorname{End}_{\mathbb{F}}(U)$ as an associative algebra, so that $a \in Z\left(\operatorname{End}_{\mathbb{F}}(U)\right) \cap \mathrm{SL}(U)=\left\langle\mathrm{i}_{U}\right\rangle$. Therefore, $\phi_{a, 1} \in\left\langle\theta=\phi_{\mathrm{i}_{U}, 1}\right\rangle$.

Equation (6.1) gives $Q /\langle\theta\rangle \cong \pi_{V} \circ \Psi(Q)$, so that the grading on $\mathfrak{s o}(V, q)$ and the $\mathbb{Z}_{4}$-grading determine the fine grading on $\mathfrak{e}_{8}$ induced by $Q$. As $\tilde{Q}=\pi_{V} \circ \Psi(Q) \subset$ $\operatorname{PSO}(V, q) \simeq \operatorname{Aut}(\mathfrak{s o}(V, q))^{\circ}$ and the grading $\Gamma^{\prime}$ induced on $\mathfrak{s o}(V, q)$ has neutral component, we can apply to $\tilde{Q}$ all the facts in remark 6.4 . In particular, $\tilde{Q}$ is isomorphic to $\mathbb{Z}_{2}^{m}$ with $m \leqslant 8$, and hence $Q$ is isomorphic to $\mathbb{Z}_{4} \times \mathbb{Z}_{2}^{m}(4 \leqslant m \leqslant 8)$. We will see that $m=6$.

The commutativity of $Q$ gives $\phi_{x_{1} y_{1}, p_{1} \cdot q_{1}}=\phi_{y_{1} x_{1}, q_{1} \cdot p_{1}}=\phi_{-x_{1} y_{1}, q_{1} \cdot p_{1}}$ and we get $p_{1} \cdot q_{1}=-q_{1} \cdot p_{1}$. In this way, we check that the elements $p_{1}, q_{1}, p_{2}, q_{2}$ in $\operatorname{Spin}(V, q) \subseteq$ $C(V, q)_{\overline{0}}$ satisfy the same commutation relations as $x_{1}, y_{1}, x_{2}, y_{2}$. In particular, the elements $p_{i}, q_{i}$ are not in $Z(\operatorname{Spin}(V, q))=\langle z\rangle$. Besides, $\operatorname{Ad}_{p_{1}} \in\left\langle\operatorname{Ad}_{e_{i} \cdot e_{j}}: i \neq j\right\rangle$, and $\theta \phi_{x_{1}, p_{1}}=\phi_{1_{U}, z} \phi_{x_{1}, p_{1}}=\phi_{x_{1}, z \cdot p_{1}}, \theta^{2} \phi_{x_{1}, p_{1}}=\phi_{x_{1},-p_{1}}$. Hence we may replace $p_{1}$ by $z \cdot p_{1}$ or by $-p_{1}$, and the same for $p_{2}, q_{1}, q_{2}$. Therefore, we may assume that each $p_{i}$ or $q_{i}$ is of the form $e_{i} \cdot e_{j}$ for $i \neq j$, or $e_{i} \cdot e_{j} \cdot e_{k} \cdot e_{l}$, for different $i, j, k, l$.

Then $Q$ is generated by $\phi_{x_{1}, p_{1}}, \phi_{y_{1}, q_{1}}, \phi_{x_{2}, p_{2}}, \phi_{y_{2}, q_{2}}$ and by the elements in $Q$ of the form $\phi_{1_{U}, s}$ with $s \in \operatorname{Spin}(V, q)$. Moreover, these elements $s$ belong to $\left\{ \pm e_{i_{1}} \cdot e_{i_{2}}\right.$. $\cdots e_{i_{r}}: 1 \leqslant i_{1}<\cdots<i_{r} \leqslant 10, r$ even, $\left.r \neq 2, r \neq 8\right\}$ by lemma 6.2(a). Besides this, the commutativity of $Q$ ensures that any element $s \in \operatorname{Spin}(V, q)$ such that $\phi_{1_{U}, s} \in Q$ commutes with $p_{1}, q_{1}, p_{2}, q_{2}$, and any two elements $s, s^{\prime} \in \operatorname{Spin}(V, q)$ with $\phi_{1_{U}, s}, \phi_{1_{U}, s^{\prime}} \in Q$ commute. We are going to reduce the task of determining $Q$ to an easy combinatorial problem.

Given two sequences $I=\left(i_{1}, \ldots, i_{r}\right)$ and $J=\left(j_{1}, \ldots, j_{s}\right)$, with $1 \leqslant i_{1}<\cdots<$ $i_{r} \leqslant 10,1 \leqslant j_{1}<\cdots<j_{s} \leqslant 10$, consider the elements $e_{I}:=e_{i_{1}} \cdot e_{i_{2}} \cdots e_{i_{r}}$, $e_{J}:=e_{j_{1}} \cdot e_{j_{2}} \cdots e_{j_{s}}$ in $C(V, q)$ as above. Then $e_{I} \cdot e_{J}=(-1)^{|I \cap J|} e_{J} \cdot e_{I}$.

Identify $I$ with the element $x_{I} \in \mathbb{Z}_{2}^{10}$ with $\overline{1}_{\mathrm{s}}$ in the components $i_{1}, \ldots, i_{r}$ and $\overline{0} \mathrm{~s}$ elsewhere, and similarly for $J$. Then $e_{I} \cdot e_{J}=(-1)^{x_{I} \bullet x_{J}} e_{J} \cdot e_{I}$, where, for elements $x, y \in \mathbb{Z}_{2}^{10}, x \bullet y=\sum_{i=1}^{10} x_{i} y_{i}$ denotes the natural symmetric (and at the same time alternating) non-degenerate bilinear form on $\mathbb{Z}_{2}^{10}$. In other words, the elements $e_{I}$ and $e_{J}$ commute in $C(V, q)$ if and only if $x_{I}$ and $x_{J}$ are orthogonal in $\mathbb{Z}_{2}^{10}$.

Note that $z=e_{1} \cdot e_{2} \cdots e_{10}=e_{(1,2,3, \ldots, 10)}$. Let $K=\mathbb{Z}_{2}(\overline{1}, \overline{1}, \ldots, \overline{1})$. Then $K^{\perp}$ is the subspace of the elements $x_{I}$ with $|I|$ even (i.e. $e_{I} \in \operatorname{Spin}(V, q)$ ). The bilinear form $\bullet$ induces a non-degenerate alternating bilinear form on $K^{\perp} / K$.

Then the problem of finding a maximal finite abelian subgroup $Q$ under the conditions above is equivalent to the problem of finding maximal subspaces $S$ of $K^{\perp} / K$ that are the orthogonal sum of two orthogonal hyperbolic planes $U_{1}$ and $U_{2}$ (corresponding to $\left\{p_{1}, q_{1}\right\}$ and $\left\{p_{2}, q_{2}\right\}$ ), and a totally isotropic subspace $U_{3}$ orthogonal to $U_{1}$ and $U_{2}$. That is, $S=U_{1} \perp U_{2} \perp U_{3}, U_{i} \bullet U_{i} \neq 0, i=1,2$, 
$U_{3} \bullet U_{3}=0, U_{i} \bullet U_{j}=0$ for $i \neq j$, and $\operatorname{dim}_{\mathbb{Z}_{2}} U_{1}=\operatorname{dim}_{\mathbb{Z}_{2}} U_{2}=2$, and with the extra condition that there is no $\bar{x} \in U_{3}$ ( $\bar{x}$ denotes the class modulo $K$ of an element $\left.x \in \mathbb{Z}_{2}^{10}\right)$ with $|\operatorname{Supp}(x)|=2$ or 8 , where $\operatorname{Supp}(x)$ is the set of indices with $x_{i} \neq \overline{0}$.

Since $\operatorname{dim}_{\mathbb{Z}_{2}} K^{\perp} / K=8$, the maximal dimension for $U_{3}$ is 2 and in this case $S=U_{3}^{\perp}$. As above, let $\left\{\varepsilon_{1}, \ldots, \varepsilon_{10}\right\}$ be the canonical basis of $\mathbb{Z}_{2}^{10}$. Up to reordering of indices, the only 'maximal' possibility is given by

$$
U_{3}=\mathbb{Z}_{2}\left(\overline{\varepsilon_{1}+\varepsilon_{2}+\varepsilon_{3}+\varepsilon_{4}}\right) \oplus \mathbb{Z}_{2}\left(\overline{\varepsilon_{3}+\varepsilon_{4}+\varepsilon_{5}+\varepsilon_{6}}\right) .
$$

(Note $\mathbb{Z}_{2}\left(\overline{\varepsilon_{1}+\varepsilon_{2}+\varepsilon_{3}+\varepsilon_{4}}\right) \oplus \mathbb{Z}_{2}\left(\overline{\varepsilon_{5}+\varepsilon_{6}+\varepsilon_{7}+\varepsilon_{8}}\right)$ is not valid, as $\overline{\varepsilon_{1}+\cdots+\varepsilon_{8}}=$ $\overline{\varepsilon_{9}+\varepsilon_{10}}$, which is the class, modulo $K$, of an element with support of size 2 .)

Then, up to a reordering of indices, there is a unique maximal possibility:

$$
S=U_{3}^{\perp}=\operatorname{span}\left\{\overline{\varepsilon_{1}+\varepsilon_{2}}, \overline{\varepsilon_{3}+\varepsilon_{4}}, \overline{\varepsilon_{5}+\varepsilon_{6}}, \overline{\varepsilon_{1}+\varepsilon_{3}+\varepsilon_{6}+\varepsilon_{7}}, \overline{\varepsilon_{8}+\varepsilon_{9}}, \overline{\varepsilon_{9}+\varepsilon_{10}}\right\} .
$$

\section{Acknowledgements}

C.D. was supported by the Spanish Ministerio de Economía y CompetitividadFondo Europeo de Desarrollo Regional (FEDER) Grant nos MTM2013-41208-P and MTM2016-76327-C3-1-P, and by the Junta de Andalucía (Grant nos FQM-336 and FQM-7156), with FEDER funds. A.E. was supported by the Spanish Ministerio de Economía y Competitividad-Fondo Europeo de Desarrollo Regional (FEDER) Grant no. MTM2013-45588-C3-2-P, and by the Diputación General de AragónFondo Social Europeo (Grupo de Investigación de Álgebra).

\section{References}

1 Y. A. Bahturin and M. Tvalavadze. Group gradings on $G_{2}$. Commun. Alg. 37 (2009), 885-893.

2 C. Draper. A non-computational approach to the gradings on $\mathfrak{f}_{4}$. Rev. Mat. Ibero. $\mathbf{2 8}$ (2012), 273-296.

3 C. Draper and C. Martín-González. Gradings on $\mathfrak{g}_{2}$. Linear Alg. Applic. 418 (2006), 85111.

4 C. Draper and C. Martín-González. Gradings on the Albert algebra and on $\mathfrak{f}_{4}$. Rev. Mat. Ibero. 25 (2009), 841-908.

5 C. Draper and A. Viruel. Fine gradings on $\mathfrak{e}_{6}$. Publ. Mat. 60 (2016), 113-170.

6 A. Elduque. Jordan gradings on exceptional simple Lie algebras. Proc. Am. Math. Soc. 137 (2009), 4007-4017.

7 A. Elduque. Fine gradings on simple classical Lie algebras. J. Alg. 324 (2010), 3532-3571.

8 A. Elduque. Fine gradings and gradings by root systems on simple Lie algebras. Rev. Mat. Ibero. 31 (2015), 245-266.

9 A. Elduque and M. Kochetov. Gradings on the exceptional Lie algebras $F_{4}$ and $G_{2}$ revisited. Rev. Mat. Ibero. 28 (2012), 773-813.

10 A. Elduque and M. Kochetov. Gradings on simple Lie algebras, Mathematical Surveys and Monographs, vol. 189 (Providence, RI: American Mathematical Society, 2013).

11 A. Elduque and M. Kochetov. Graded modules over classical simple Lie algebras with a grading. Israel J. Math. 207 (2015), 229-280.

12 A. Elduque and M. Kochetov. Gradings on the Lie algebra $D_{4}$ revisited. J. Alg. 441 (2015), 441-474.

13 R. L. Griess Jr. Elementary abelian $p$-subgroups of algebraic groups. Geom. Dedicata 39 (1991), 253-305. 
14 G. Han and D. A. Vogan Jr. Finite maximal tori. In Symmetry: representation theory and its applications, Progress in Mathematics, vol. 257, pp. 269-303 (Springer, 2014).

15 J. E. Humphreys. Linear algebraic groups, Graduate Texts in Mathematics, vol. 21 (Springer, 1975).

16 J. E. Humphreys. Conjugacy classes in semisimple algebraic groups, Mathematical Surveys and Monographs, vol. 43 (Providence, RI: American Mathematical Society, 1995).

17 V. G. Kac. Infinite-dimensional Lie algebras, 3rd edn (Cambridge University Press, 1990).

18 A.-L. Onishchik and E.-B. Vinberg. Lie groups and Lie algebras III: structure of Lie groups and Lie algebras, Encyclopaedia of Mathematical Sciences, vol. 41 (Springer, 1994).

19 J. Patera and H. Zassenhaus. On Lie gradings. I. Linear Alg. Applic. 112 (1989), 87-159.

20 J. Yu. Maximal abelian subgroups of compact simple Lie groups. Preprint, 2012. (Available at https://arxiv.org/abs/1211.1334.)

21 J. Yu. Maximal abelian subgroups of compact simple Lie groups of type E. Geom. Dedicata 185 (2016), 205-269. 